\title{
Stabil gelagert bis zum Tod?
}

Die stabile Seitenlage kann es erschweren, einen Atemstillstand rasch zu erkennen und mit einer Herzdruckmassage zu beginnen, wie spanische Forscher berichten.

$\mathrm{Zu}$ den wichtigsten Maßnahmen in der Notfallversorgung bewusstloser Patienten gehört es, das Versagen der Atmung zu erkennen und in diesem Fall sofort mit der Herzdruckmassage zu beginnen. Hierfür wird in den Leitlinien zur Reanimation des European Resuscitation Council empfohlen, den Patienten in die HTCL(Head Tilt Chin Lift)-Position zu bringen: „Legen Sie Ihre Hand auf seine Stirn und ziehen Sie seinen Kopf leicht nach hinten; Heben Sie mit Ihren Fingerspitzen das Kinn des Patienten an, um die Atemwege frei zu machen. Drehen Sie den Patienten auf den Rücken.“

Andererseits gilt bei bewusstlosen, spontan atmenden Patienten die stabile
Seitenlagerung als optimal, um eine Aspiration zu verhindern. Was aber, wenn die zunächst spontane Atmung sistiert? Lässt sich ein sich anbahnender Atemstillstand hier gegebenenfalls rasch identifizieren?

„Nein“, meint eine Forschergruppe um den Notfallspezialisten Miguel FreireTellado (Lugo, Spanien). Die Wissenschaftler hatten in einer Studie 59 Teilnehmer, die in Herz-Lungen-Wiederbelebung ausgebildet waren, in zwei Gruppen eingeteilt: Eine hatte gelernt, die Patienten in stabile Seitenlage zu bringen, die zweite in die HTCL-Lagerung.

Freire-Tellado und Kollegen stoppten, wie schnell die Probanden in einer simulierten Notfallsituation den einsetzenden
Atemstillstand eines Bewusstlosen bemerkten. Binnen zwei Minuten schafften das 14 von 27 Studienteilnehmern bei Seitenlagerung und 23 von 28 bei Lagerung in HTCL-Position.

\section{Schlechtere Einschätzung der Atemtätigkeit}

„Die stabile Seitenlage behinderte die Einschätzung der Atemtätigkeit und verzögerte das Erkennen des Atemstillstands", fassen Freire-Tellado und Mitarbeiter die Resultate ihrer Studie zusammen. Im Vergleich zur HTCL-Position habe dies den Beginn der Herzdruckmassage verzögert und die Wahrscheinlichkeit erhöht, nicht mit der kardiopulmonalen Reanimation zu beginnen.

Freire-Tellado M et al. Resuscitation 2017, online 9. April; https://doi.org/10.1016/j.resuscitation.2017.03.008

\section{Lebensgefährlich oder nicht?}

\section{Mit Thoraxschmerz zum Hausarzt: Was dahinter steckt}

Das Risiko einer lebensbedrohlichen Ursache von Thoraxschmerz wird von Hausärzten häufig überund selten unterschätzt.

Wenn Patienten in der Praxis über Schmerzen im Brustbereich klagen, wollen Hausärzte kein Risiko eingehen. Bei etwa jedem sechsten Patienten lautet die Verdachtsdiagnose akutes Koronarsyndrom (ACS). Tatsächlich sind aber nicht einmal halb so viele Patienten davon betroffen. Dass eine lebensbedrohliche Ursache übersehen wird, kommt selten vor. Das hat eine Studie in 118 Hausarztpraxen in den Niederlanden und Belgien ergeben, bei der zwei Wochen lang alle Diagnosen prospektiv erfasst wurden.
281 von insgesamt 22.294 Patientenkontakten $(1,26 \%)$ waren durch Schmerzen im Brustbereich veranlasst. Bei 17,7\% stellten die Hausärzte die Arbeitsdiagnose einer lebensbedrohlichen kardialen Erkrankung. Letztlich wurde dieser Verdacht bei 6,5\% der Patienten bestätigt. Eine lebensbedrohliche Ursache wurde bei insgesamt $8,4 \%$ (von 263 Patienten mit bekannter Abschlussdiagnose) gefunden: 17 Patienten mit ACS, vier mit stabiler KHK und einer mit Lungenembolie.

\section{Meist muskuloskeletale Ursachen}

Die häufigste Arbeitsdiagnose war muskuloskeletal bedingter Schmerz (26,1\%). Dieser stand auch bei den endgültigen Diagnosen einen Monat später auf Platz 1
(33,1\%). Bei den Abschlussdiagnosen folgten psychische Beschwerden inklusive Hypoventilation $(17,1 \%)$ und gastrointestinale Störungen $(13,2 \%)$.

Unterschiede zwischen Hausarzt- und finaler Diagnose, die Konsequenzen für die Behandlung haben könnten, gab es bei 23\% der Patienten. In den meisten Fällen hatte der Hausarzt eine lebensgefährliche kardiovaskuläre Ursache vermutet, obwohl eine harmlosere Erkrankung dahinter stand. In zwei Fällen (0,7\%) hatte der Hausarzt das Risiko jedoch unterschätzt und eine ischämische Herzerkrankung als Vorhofflimmern und einen Myokardinfarkt als Pneumonie verkannt. 